\title{
Cost Efficient Fast Autonomous Reconfiguration System in Wireless Mesh Networks
}

\author{
N.N.Krishnaveni \\ Department of Computer science \\ Research Scholar, Bharathiar University \\ Coimbatore, India \\ Dr.K.Chitra \\ Department of Computer science \\ Asst.Professor, Govt.Arts College \\ Melur, Madurai
}

\begin{abstract}
While comparing with the existing networks, Wireless Mesh Network has the advantages of fast implementation, low direct investment and easy maintenance. During their existence time Wireless Mesh Networks (WMNs) experience frequent link failures caused by channel interference, dynamic obstacles, and/or applications bandwidth demands. Wireless mesh networks should be recovered from these link and node failures. These failures cause severe performance degradation in WMNs. In WMNs, the quality of the link can rapidly change because of varying environment condition. The routing algorithm must be able to cope with such changes in link quality and provide alternate route in case the link becomes unusable.

This paper proposes a Cost efficient fast autonomous reconfiguration system (C-FARS) which provides the multiradio Wireless Mesh Networks to recover from link failure automatically to maintain the network performance. C-FARS generates necessary changes in local radio and channel allocations in order to recover from failures. C-FARS recovers from link failures by making cost effective local configuration changes with minimum delay that satisfies the applications QoS demands instead of making global network changes. Our Implementation results shows that C-FARS outperforms existing failure-recover schemes in improving channel-efficiency by more than $95 \%$, also reduces the cost and delay.
\end{abstract}

Keywords- Wireless Mesh Networks (WMNs); Cost efficient autonomous reconfiguration system(C-FARS); WMN Architecture, Quality of service $(Q o S)$.

\section{INTRODUCTION}

\section{A. Wireless Mesh Networks Architecture}

Wireless mesh networking has emerged as a promising design paradigm for next generation wireless networks. WMNs seem significantly attractive to network operators for providing new applications that cannot be easily supported by other wireless technologies. A WMN is formed by a set of wireless nodes, where each node can communicate and forward data of each other. Wireless Mesh Network consists of two types of nodes: mesh clients (MCs), mesh routers (MRs). Some of the mesh routers act as gateways to the internet using the wired links. These special WMRs are called Internet Gateway (IGWs). During communication the mesh networks divide the long distance into a series of smaller hops to boost the signal using intermediate nodes. Such structural design allows continues flow of data and reconfiguration when paths are blocked or broken. WMN is fully supported by wireless mesh router network, also called Backbone Wireless Mesh Network (BWMNs). IGWs acts as communication bridges between the internet and BWMN, and provides internet accessibility. These BWMNs provide internet connectivity to MCs. 


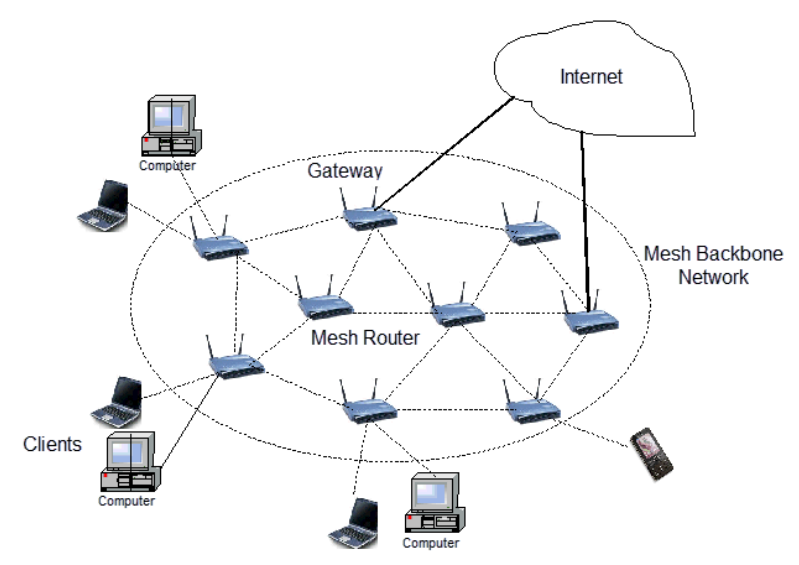

Fig. 1. WMNs architecture

\section{B. Why Is Self-Reconfigurability Necessary?}

Wireless Mesh Network has the advantages of fast implementation, easy maintenance and low direct investment while comparing with the existing networks. During their life time Wireless Mesh Networks (WMNs) experience frequent link failures caused by channel interference, dynamic obstacles, and/or applications bandwidth demands. Wireless mesh networks should be recovered from these link and node failures. These failures cause severe performance degradation in WMNs.

The topology and the connectivity of the network can vary frequently because of route failure and energy depletions; an efficient self-configuration, topology control and power managements are required. In WMNs, the quality of the link can rapidly change because of varying environment condition. The routing algorithm must be able to cope with such changes in link quality and provide alternate route in case the link becomes unusable. This approach gives the research on the fault-tolerance for Wireless mesh networks

\section{Techniques For Link Recovery In WMN}

Though MWNs are used widely, they often face frequent link failures. So link recovery plays an important role in WMN. There are various techniques used for link recovery in WMN.

1) Initial Resource Allocation Method: In this method, using some theoretical guidelines for allocation of resources, the initial planning is done to recover from link failure. Even the planning is done before, this method has drawback of "Global reconfiguration changes". By using this method for link recovery where a small change is required, this method performs reconfiguration of the entire network [3].

2) Greedy Channel Assignment Method: In this method the drawback of "Initial Resource Allocation Method" is removed. In this method, the setting of faulty link is alone changed instead of the entire network. Even it do local changes it too has drawback of "ripple effect". In this effect whenever a local change is done then it causes triggering of change to some kind of network settings [4].

3) Fault Tolerant Routing Protocol: When there is a link failure during the communication in WMN, then it is must to recover the link from failure and also make sure that the communication must not loss. To overcome these link failures and avoid the data loss we can route the packet through different link. Protocols help us in selecting the alternate paths. Protocols such as rerouting or multipath routing can be used. Anyway these protocols are not that much efficient and take massive amount of time to reroute, which leads to delay delivery of packets.

4) Autonomus Reconfiguration System (ARS): Kim and Shin [5] proposed a new recovery technique for wireless mesh networks named ARS. In this method the node detects the link failure and generates a set of reconfiguration plans by considering the rage of channels. Among the set of plans, a feasible plan is selected by considering that the plan must maximizes the throughput of the network and also satisfy some QoS constraint of the network. The drawback of ARS is that it is not cost aware reconfiguration technique.

\section{5) Enhanced Reconfiguration System (ERS):} Ramakrishnan R and Dr. N.Sankar introduces ERS [6] is to provide cost aware reconfiguration system in wireless mesh networks. In ERS a set of reconfiguration plans are generated and among them a best plan is selected which provides the required service in minimal cost. According to the selected plan the reconfiguration is done in the network. Thus it maximizes the network performance. The drawback of ERS is, sometimes it may fail to satisfy the Qos constraint which leads to frequent link failure.

6) Quick Autonomous Reconfiguration System (QARS): QARS [7] is proposed by A.Melveena \& D.Ramya Dorai to recover the link failure in a short duration to avoid the delay transmission. In this method when a link failure is detected, immediately a group is formed around the faulty area. Among the group, a leader node is selected using enhanced Bully algorithm. Using the plans generated by other nods in the group, the leader node select the plan which requires minimum change and meets the Qos demand. Thus it improves the network performance.

\section{C-FARS ARCHITECTURE AND ALGORITHM}

\section{A. C-FARS Architecture}

In the proposed system a network is assumed to consist of mesh nodes, IEEE 802.11 based wireless links and control gateways as in fig. 2 . 


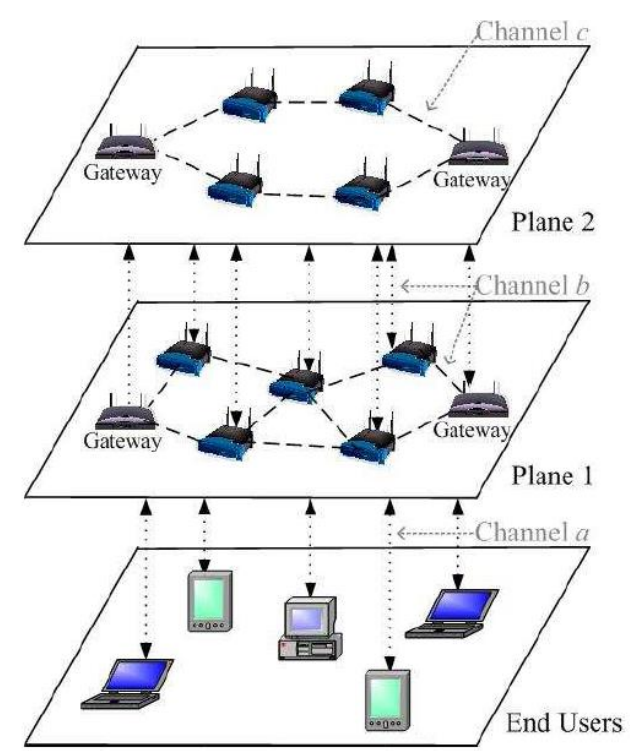

Fig. 2. C-FARS architecture

C-FARS running in every mesh node supports selfreconfigurability via the following distinct features:

- Localized Reconfiguration: Based on the multiple channels and radio associations available, C-FARS generates reconfiguration plans that allow for only local network changes where the link failures occurred.

- Fast Reconfiguration: C-FARS generates the reconfiguration plans within minimum duration and reduces the delay in reconfiguration. Such fast reconfiguration helps in increasing network performance.

- Qos-Aware Planning: C-FARS effectively identifies QoS-satisfiable reconfiguration plans by : 1) Estimating the QoS-Satisfiability of generated reconfiguration plans, and 2) Deriving their expected benefits in channel utilization.

- Cross-Layer Interactions: C-FARS actively interacts across the network and link layers for planning. This interaction enables C-FARS to include rerouting for reconfiguration planning in addition to link-layer reconfiguration. C-FARS also maintains connectivity during the recovery period with the help of a routing protocol.

- Cost Effective Multipath Selection: The routing algorithm must be able to cope with changes in link quality and rapidly provide an alternative route in case the link becomes unusable. Our modified DSR algorithm in C-FARS selects the least cost alternative path based on various routing metrics among the multiple paths available in case of path failure.

\section{B. C-FARS Algorithm}

In this paper we proposed an algorithm for C-FARS which operates on each and every node of the Wireless Mesh Networks (WMNs). This algorithm1 executes in a particular time interval to monitor the failure and if found it recovers from such failure in an efficient manner.

Using this algorithm it recovers from the link failure and if there is a frequent link failure detected on the same link which means the particular link unusable, then it is consider as a path failure and a new cost effective path is selected using modified DSR.

(1) Monitoring period

1: for every link do

2: measure link-quality using passive monitoring;

3: end for

4: send monitoring results to a gateway ;

(2) Failure detection and group formation period

5: if link violates link requirements then

6: request a group formation on channel of

link;

7: end if

received;

8: participate in a leader election if a request is

(3) Planning period

9: if node is elected as a leader then 10: send a planning request message to a gateway;

11: else if node is a gateway then

12: synchronize requests from

reconfiguration groups

13: generate a reconfiguration plan;

15: end if

14: send a reconfiguration plan to a leader;

(4) Reconfiguration period

16: if includes changes of node then

18: end if

17: apply the changes to links;

19: relay to neighboring members, if any

Algorithm. 1.

C-FARS algorithm 1 involves 4 phases

1) Network Monitoring: In this 1st phase each mesh node monitors the quality of its outgoing wireless links at every tm $\mathrm{sec}(\mathrm{eg} .10 \mathrm{sec})$ and the status of the results are reported to the gateway. 
2) Failure Detection Phase And Group Formation: System detects the failure by comparing the current link state information with that in the existing database. Whenever a failure is detected all the mesh nodes that use a faulty channel are grouped together.

3) Planning Period: After forming a group one of the group members will be elected as a leader by using the wellknown bully algorithm and this leader node will send a plan request message to the gateway. Now the gateway generates the new reconfiguration plan which is broken down into 3 steps as in fig. 3.

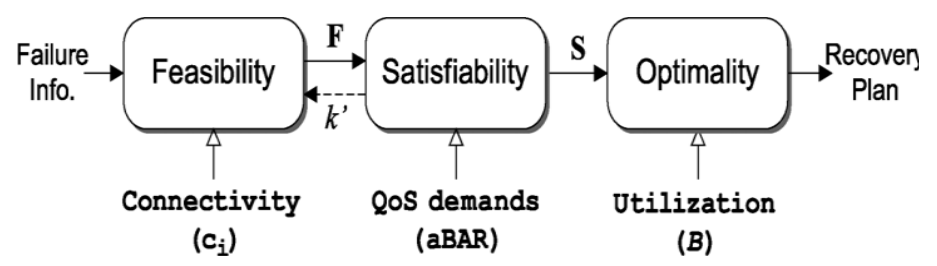

Fig. 3. Steps in planning period

a) Feasible Plan Generation: Generating feasible plans is very important to keep in mind all legitimate changes in links configurations and their combinations around the faulty area. C-FARS identifies feasible changes that do only local changes but maintain existing network connectivity as much as possible. While selecting feasible plan C-FARS will 1) avoid a faulty channel. 2) Maintain network connectivity and utilization. 3) Control the scope of reconfiguration changes.

b) QoS-Satisfiability Evaluation: Among a set of feasible plans, C-FARS filters the plan that satisfying QoS by checking if the QoS constraints are met under each plan. CFARS will select the plan by 1)Per-link bandwidth estimation 2) Examining per-link bandwidth satisfiability 3) Avoiding cascaded link failures

c) Choosing the Best Plan: C-FARS now has a set of reconfiguration plans that are QoS-satisfiable and needs to choose a plan within the set for a local network to have evenly distributed link capacity. C-FARS selects the plan by 1) Quantifying the fairness of a plan 2) Path with the highest minimum access efficiency value 3) Breaking a tie among multiple plans

4) Implementing Reconfiguration Plan: Here firstly the gateway sends the selected reconfiguration plan to the leader node then leader node distributes it to all other nodes in the group and then each node executes the corresponding configuration changes

\section{PATH SELECTION IN C-FARS}

C-FARS is proposed to overcome the failure by making the reconfiguration with minimum delay and less expensive. Once a node detects the failure, it self-reconfigures by using feasible plans. If there is no feasible plan for a particular link failure or it detects frequent failure on a particular link, an alternate route must be discovered. The new route selected must be cost effective, feasible, optimal, and satisfies QoS demands.

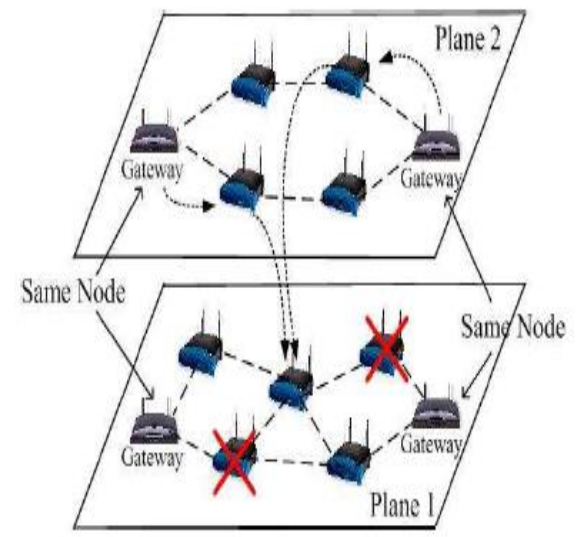

Fig. 4. C-FARS Failure and reconfiguration

\section{A. Route Discovery Using Modified DSR}

The rationale for modifying the DSR protocol is to make it better suited to the WMN environment. The WLAN medium is a shared medium where nodes must contend for accessing the medium using DCF MAC mechanism. Since the DCF is a "listen before talk mechanism", a high level of contention for access to the medium will result in a low availability of bandwidth at a node. This in turn limits the maximum throughput that can be achieved. Unfortunately, the DSR protocol fails to explicitly consider the availability of bandwidth locally at a node which is an important omission in WMNs based upon the IEEE 802.11 standard.

In this case, the access efficiency measured locally at a node is used as a measure of the local availability of bandwidth at that node. By incorporating bandwidth availability information into the DSR protocol the cost of Route Discoveries can be reduced and the overall performance of the network can be significantly improved. The performance of modified DSR is investigated through a series of simulations performed on the NS2 modeler package.

In this work a new metric to support the DSR node cache mechanism has been used. The modification to the DSR protocol is intended to incorporate knowledge of the path capacity into the route discovery mechanism. Specifically, the DSR protocol was modified by replacing hop count field in the cache route table with an access efficiency field. The 
optimal route is determined by selecting the path with the highest minimum access efficiency value.

Fig. 5. Illustrates the operation of the path selection mechanism for the DSR protocol and our modified DSR protocol. From this figure, the original DSR protocol selects path B as the hop count of this path is smaller than the hop count of the other paths (path $\mathrm{A}$ and path $\mathrm{C}$ ). While our modified DSR protocol chooses path A over paths B and C as it selects the path with the highest minimum link capacity.

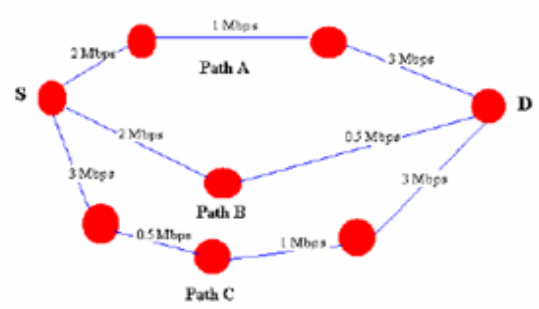

Fig. 5. Path selection in C-FARS

\section{B. Path Selection Metric}

In this section we present a new interference-aware multipath selection metric. Our selection metric and algorithm aims to minimize interference (between paths and to neighbor nodes), without assuming that interference is global, and considers link quality and the delaying effect of long paths, when selecting paths.

1) Path Interference Cost: The path interference cost reflects the degree of interference between links operating on a common channel along the selected paths. To help define the path interference cost, we first define the interference cost for a link $(i, j)$ on channel $c$ in a network $N$ as:

$$
L I_{i j}(c, N)=E T T_{i j}(c) * S_{i j}(c, N),
$$

Where $S_{i j}(c, N)$ denotes the number of nodes in network $N$ that are affected by interference from link $(\mathrm{i}, \mathrm{j})$ on channel $c$. In this work we assume interference to be binarytwo nodes either do or do not interfere. This assumption allows us to simplify the computation of metrics. We plan to investigate more accurate classifications of interference in the future. The path interference cost for a set of paths $P$ is simply the aggregate of all link interference costs along the paths:

$$
P I C_{P}=\sum_{i j \in p} L I_{i j}(c, P)
$$

2) Neighbour Interference Cost: Similar to the path interference cost, the neighbor interference cost represents the channel time cost to nodes close to the paths, and it is defined as:

$$
N I C_{P}=\sum_{i j \in p} L I_{i j}(c, N-P),
$$

3) Weighted Interference Multipath metric: The Weighted Interference Multipath (WIM) metric is a weighted average of path interference and the neighbor interference costs.

$$
\begin{aligned}
& \qquad \operatorname{WIM}_{P}=\beta * N I C_{P}+(1-\beta) * P I C_{P}, \\
& \text { where the parameter } \beta \text { satisfies } 0 \leq \beta \leq 1 .
\end{aligned}
$$

The WIM metric can be interpreted as a balance between local and global considerations. The path interference cost component reflects the total channel time along the paths that is consumed when the channels are concurrently used. The smaller it is, the better the paths will be at providing low endto-end delay, assuming interference to neighbor nodes is negligible. On the other hand, the neighbor interference component favors paths that have less interference to nodes that are not on the paths. This could be beneficial when the network load is high such that interference from neighboring nodes starts to affect, or even dictate, the traffic on the paths.

\section{C-FARS Fault Tolerance}

Physical C-FARS can play very significant role for failure recovery in a WMN. As any node in plane 1 is reachable from plane 2 in minimum hops, if any node on plane 1 fails, all the data destined to that node can be redirected over plane 2 . Moreover, because of the ring architecture, if any node on $\mathrm{C}$ FARS suffers a failure, data can still be routed over other part of the C-FARS. As all the gateways of plane 1 is included in plane 2 , if a gateway fails, then traffic can be redirected to the other gateway, and C-FARS can carry the traffic from the other gateway, and deliver the packets. Failure recovery schemes described can be integrated with C-FARS for protection. Fig. 4. shows how C-FARS can be used for fault tolerance in a WMN.

\section{Energy Efficient Model}

The topology control problem is a well researched topic for energy saving in wireless ad hoc networks. However, little attention has been given to similar problems in the case of wireless mesh networks (WMNs) even though WMNs have very unique characteristics that are different from other wireless multihop networks e.g., MANETs. This is because many WMN surveys make the impractical assumption that since mesh routers are static, energy is not a problem.

Consequently, with specific interests to WMN applications in rural areas, where power sources are limited, this work addresses the topology control problem for energy efficiency in a hybrid WMN of heterogeneous wireless devices with 
varying transmission ranges. A localized distributed algorithm is presented which computes an optimal per-node transmission power such that: (1) a node's average out degree is reduced considerably to cover only the nearest neighbors, (2) network connectivity is maintained and (3) the network lifetime is extended. The performance of the algorithm is evaluated via several mathematical analyses.

Additionally, simulations are done in the NS-2 simulation environment to show correctness and effectives of the algorithm. a cross-layer modification to the DSR protocol that increases the global throughput in wireless mesh networks. In our modified DSR protocol we have introduced the Access Efficiency metric as an alternative to the hop-count metric in order to improve the route selection mechanism.

The selected path in the route selection mechanism is identified by choosing the path with the highest minimum Access Efficiency value. We have employed the NS2 modeler as a simulator to examine two different patterns of traffic for a series of randomly generated network topologies. Each topology was simulated twice. One simulation used the original DSR algorithm while the other utilized the modified DSR algorithm. The average throughput was recorded for each run and the percentage improvement for the particular topology was calculated. Our results demonstrate that a significant increase in the global throughput of the networks can be achieved by implementing our modified DSR protocol.

\section{PERFORMANCE EVALUVATION}

Our following experimental evaluations on an ns2-based simulation will demonstrates the effectiveness of C-FARS in recovering from local link-failures and in satisfying applications' diverse Qos demands

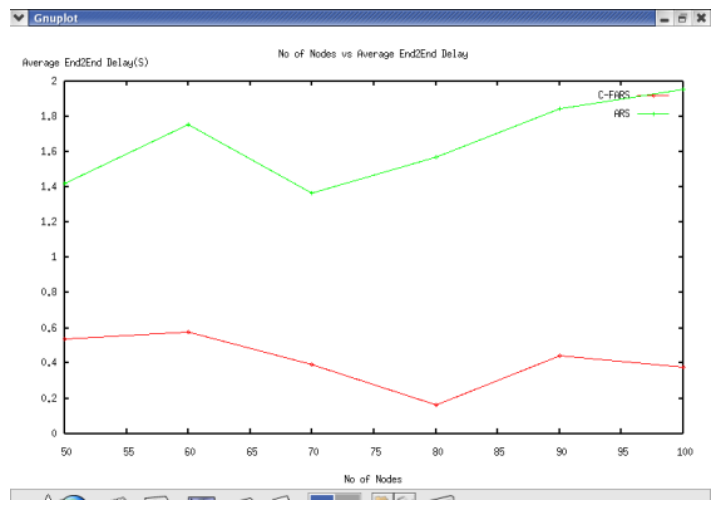

Fig. 6. Average end-to-end delay

Fig. 7.

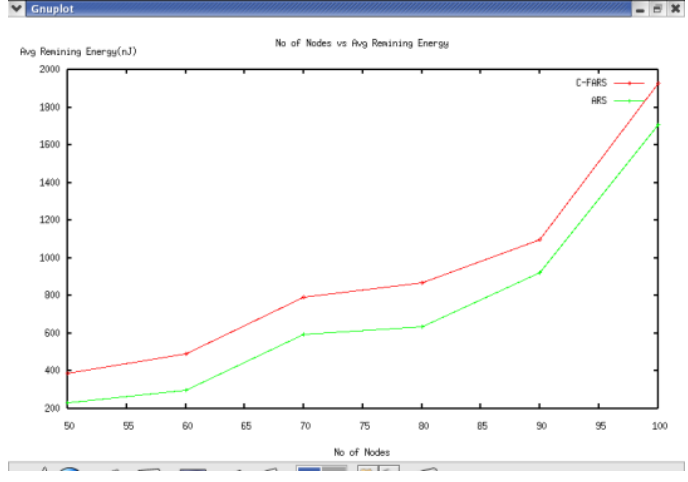

Fig. 8. Average remaining energy

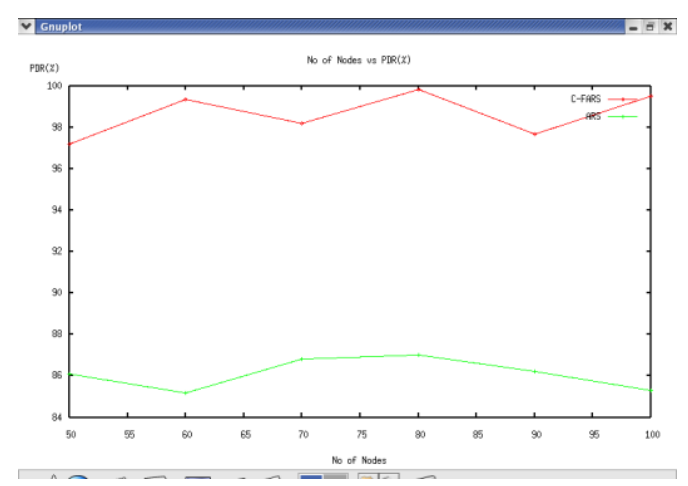

Fig. 9. Packet delivery ratio

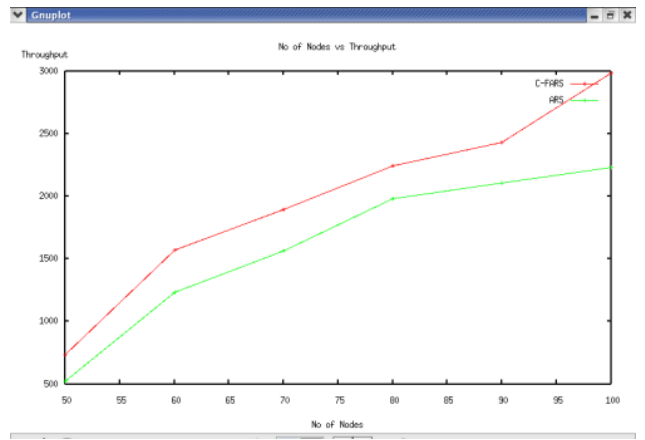

Fig. 10. Throughput

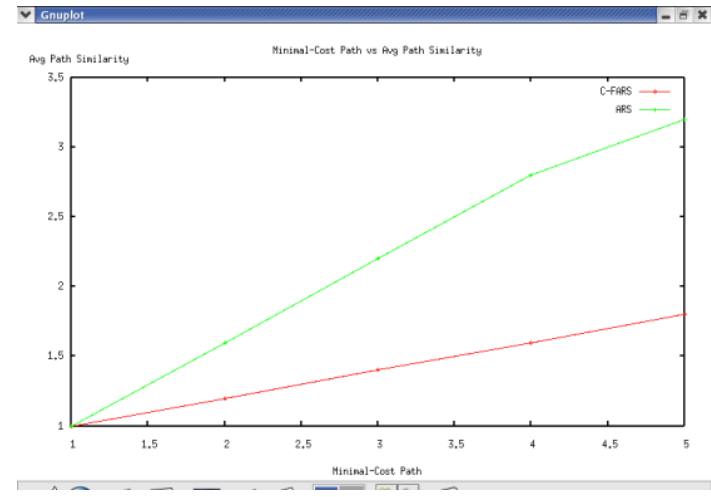

Fig. 11. Minimal cost path(path metric) 


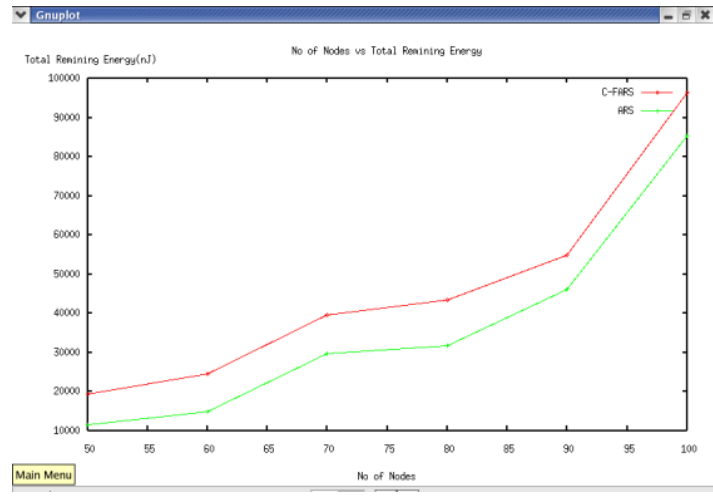

Fig. 12. Total remaining energy

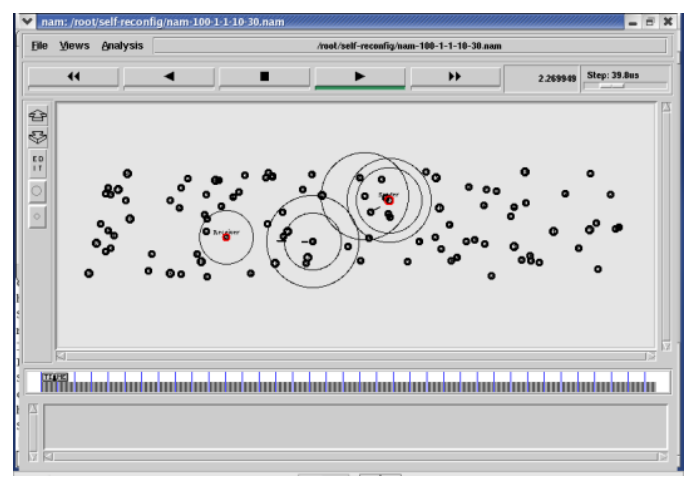

Fig. 13. Data transfer

\section{CONCLUSION}

This paper proposes a Cost efficient fast autonomous reconfiguration system (C-FARS) which provides the multiradio Wireless Mesh Networks to recover from link failure automatically to maintain the network performance. CFARS generates necessary changes in local radio and channel allocations in order to recover from failures. C-FARS recovers from link failures by making cost effective local configuration changes with minimum delay that satisfies the applications QoS demands instead of making global network changes. Our Implementation results shows that C-FARS outperforms existing failure-recover schemes in improving channelefficiency by more than $95 \%$, reduces the cost and delay.

. This scheme improves the reliability of dynamic wireless sensor networks in the point-point routing scenario by using multipath routing. This method is suitable to disseminating a large amount of bulk data to the destination with a high reliability and low delay.

Our cross-layer approach achieves the proposed reliability improvement in a dynamic wireless sensor network. Our lessons learned in this research show that in the routing protocols for WSN, optimization is more effective when taking into account the overall system and with the use of all available knowledge, instead of a strict layered approach. This approach has a much larger network lifetime, compared with traditional protocols for WMNs.

\section{REFERENCES}

[1] A.Valarmozhi, M.Subala, V.Muthu," Survey of Wireless Mesh Network", International Journal of Engineering and Innovative Technology (IJEIT) Volume 2, Issue 6, December 2012

[2] Mojtaba Seyedzadegan, Mohamed Othman, Borhanuddin Mohd Ali and Shamla Subramanian,"Wireless Mesh Networks: WMN Overview, WMN Architecture", International Conference on Communication Engineering and Networks, 2011, IPCSIT vol.19.

[3] Alicherry M, Bhatia R, and Li L, "Joint channel assignment and routing for throughput optimization in multi-radio wireless mesh networks," in Proc. ACM MobiCom, Cologne, Germany,pp. 58-72, Aug. 2005.

[4] Raniwala A and Chiueh T, "Architecture and algorithms for an IEEE 802.11-based multi-channel wireless mesh network," in Proc. IEEE INFOCOM, Miami, FL, Vol. 3, pp. 2223-2234, Mar. 2005.

[5] Kyu-Han Kim and Kang G. Shin, IEEE/ACM TRANSACTIONS ON NETWORKING, "Self-Reconfigurable Wireless Mesh Networks ", APRIL 2011

[6] Ramakrishnan R, Dr. N. Sankar Ram, Dr. Omar A. Alheyasat , “ A Cost Aware Reconfiguration Technique for Recovery in Wireless Mesh Networks", IEEE/ICRTIT , 2012

[7] A.Melveena, D.Ramya Dorai," QARS for Self Reconfiguration Mechanism in Wireless Mesh Networks", International Journal of Advanced Research in computer Engineering \& Technology (IJARCET) Volume 2, Issue 2, February 2013

[8] Kalyani Pendke 1 and S.U.Nimbhorkar," STUDY OF VARIOUS SCHEMES FOR LINK RECOVERY IN WIRELESS MESH NETWORK", International Journal on AdHoc Networking Systems (IJANS) Vol. 2, No. 4, October 2012.

[9] B.Lakshmi Sowmya1, M.Srinivas 2, B.Sravani3," A NOVEL METHOD OF DYNAMIC SELF RECONFIGURABLE WIRELESS MESH NETWORK", International Journal of Engineering Research and Development e-ISSN: 2278-067X, p-ISSN: 2278-800X, www.ijerd.com Volume 4, Issue 1 (October 2012).

[10] K.H. Kim and K. G. Shin, "On accurate and asymmetry-aware measurement of link quality in wireless mesh networks," IEEE/ACM Trans.Netw., vol. 17, no. 4, pp. 1172-1185, Aug. 2009.

[11] P. Kyasanur and N. Vaidya, "Capacity of multi-channel wireless networks: Impact of number of channels and interfaces," in Proc. ACM MobiCom, Cologne, Germany, Aug. 2005, pp. 43-57. 